\title{
Abnormal deviation in the measurement of residual urine volume using a portable ultrasound bladder scanner: a case report
}

\author{
Xueqiong $\mathrm{Zhu}^{1}{ }^{\wedge}$, Liliang $\mathrm{Zou}^{1} \wedge$, Zhao $\mathrm{Yao}^{2 \wedge}$, Zuobing $\mathrm{Chen}^{1 \wedge}$ \\ ${ }^{1}$ Department of Rehabilitation Medicine, The First Affiliated Hospital, College of Medicine, Zhejiang University, Hangzhou, China; ${ }^{2}$ The Second \\ Clinical Medical College of Wenzhou Medical University, Wenzhou, China \\ Correspondence to: Zuobing Chen. Department of Rehabilitation Medicine, The First Affiliated Hospital, College of Medicine, Zhejiang University, \\ No. 79 Qingchun Road, Shangcheng District, Hangzhou 310003, China. Email: czb1971@zju.edu.cn.
}

\begin{abstract}
In addition to being suitable for the assessment of residual urine volume, the use of a portable bladder scanner can also assist medical staff in developing a reasonable urination plan. Portable scanners can help medical personnel to estimate the height and/or relaxation of the bladder neck for pelvic floor muscle training, assist in the efficacy evaluation of muscarinic antagonists in patients with neurogenic detrusor overactivity, and even determine whether the urine in the bladder of pediatric patients meets the criteria for catheterization to obtain urine samples. However, in the case described herein, we encountered abnormal deviation between the data measured by a portal bladder scanner and actual catheterization. The patient was a 65 -year-old male suffering spinal nerve dysfunction due to tuberculous meningitis. He had functional dysuria and a history of liver cirrhosis, accompanied by a large amount of ascites. We therefore deduced that the deviation in the scanning data was attributable to the ascites. After the patient's ascites had resolved, the residual urine volume obtained via scanning evaluation was comparable to that obtained through catheterization. Our case shows that in the event of abnormal deviation in portable bladder scanner measurements, clinical judgements should be made based on the patient's condition rather than the scanning results alone.
\end{abstract}

Keywords: Portable bladder scanner; abnormal deviation; dysuria; residual urine volume; intermittent catheterization

Submitted Mar 23, 2021. Accepted for publication Jun 24, 2021.

doi: $10.21037 /$ tau-21-444

View this article at: https://dx.doi.org/10.21037/tau-21-444

\section{Introduction}

A portable bladder scanner is a medical instrument that generates multiple cross-sectional scans based on reflected sound waves. After transmission to a computer, the images obtained with a bladder scanner can be used to automatically calculate and display the bladder's capacity. Portable bladder scanners are widely used for the measurement of residual urine volume in clinical practice, due to their convenience and minimal invasiveness compared with traditional urethral catheterization (1-3). In recent years, the accuracy, efficacy, and clinical value of portable bladder scanners have been confirmed in a number of studies (4-8). However, the problems and shortcomings of using portable bladder scanners in clinical practice have not been reported in the relevant literature. Here, we briefly describe a case in our department of abnormal deviation in the measurement of residual urine volume using a portable bladder scanner.

We present the following article in accordance with the CARE reporting checklist (available at https://dx.doi. org/10.21037/tau-21-444).

\footnotetext{
^ ORCID: Xueqiong Zhu, 0000-0002-9718-9908; Liliang Zou, 0000-0003-2669-8024; Zhao Yao, 0000-0003-3016-9934; Zuobing Chen, 0000-0001-6356-3255.
} 

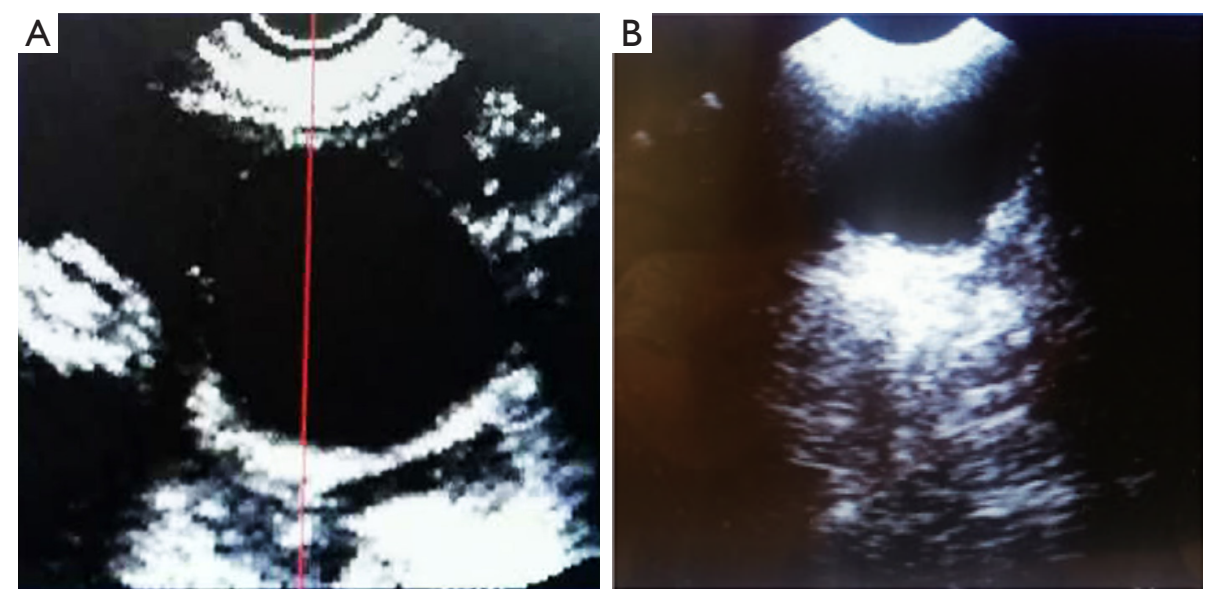

Figure 1 Effects of ascites on bladder scan results. (A) Image of the patient's bladder when ascites was present; (B) image of the patient's bladder when ascites was absent.

\section{Case presentation}

A 65 -year-old male patient was admitted to our hospital after experiencing fever with chills accompanied by fatigue for 2 days. He was admitted for treatment of lung infection and liver cirrhosis. After discharge, the patient was admitted to the Department of Rehabilitation Medicine for rehabilitative treatment due to having numbness and weakness in both lower extremities and dysuria for more than 10 days. The medical history and clinical evaluation of the patient revealed that he had suffered spinal nerve dysfunction caused by tuberculous meningitis in January 2017 and had functional dysuria. At the present, intermittent catheterization was used to assist bladder emptying for bladder training, and a portable bladder scanner was used to evaluate the residual urine volume. The patient underwent three consecutive scanning examinations to measure the residual urine volume, and the mean value was obtained. The mean residual urine volume was $560 \mathrm{~mL}$, and intermittent catheterization was implemented for bladder management. However, the actual residual urine volume was only $120 \mathrm{~mL}$. Following catheterization, the scanner still showed $440 \mathrm{~mL}$ of residual urine. Repeated measurements were performed at different time points within the next 24 hours, and all of the results were similar. Due to the tremendous difference between the residual urine volume assessed using the portable bladder scanner and the urethral catheterization results, as well as the patient's history of cirrhosis, an abdominal B-mode ultrasound examination was recommended to check for ascites. The ultrasound examination confirmed cirrhosis complicated by massive ascites (Figure 1). After clinical treatment, the patient's ascites resolved, and the residual urine volume obtained via scanning evaluation became comparable to that obtained with catheterization. Therefore, when using a portable bladder scanner to evaluate residual urine volume in the bladder, medical personnel should note that the presence of ascites may result in a serious deviation from the actual urine volume in the bladder. This study was approved by the Ethics Committee of the First Affiliated Hospital, College of Medicine, Zhejiang University. Consent for the publication of this case report was obtained from the patient's relatives.

All procedures performed in studies involving human participants were in accordance with the ethical standards of the institutional and/or national research committees and with the Helsinki Declaration (as revised in 2013).

\section{Discussion}

Major causes of dysuria include urinary tract obstruction and weak detrusor contraction. The incidence of dysuria in males is 10 -fold that in females, and it is especially prevalent in male patients over the age of 70 years, primarily because benign prostatic hyperplasia is positively correlated with age (9). In the United Kingdom, the incidence of acute urinary retention in males is approximately $3 / 1,000(10)$. For women who have given birth, the incidence of dysuria is positively correlated with age, and the incidence of dysuria after childbirth is approximately $10-15 \%$. Dysuria is seen extremely often in medical fields. In recent years, functional dysuria has attracted the attention of health care 
professionals, and portable bladder scanners have been widely used due to their unique advantages (1-8).

A portable bladder scanner is not only suitable for the assessment of residual urine volume but can also guide intermittent catheterization and assist in the development of a reasonable urination plan for patients with voiding dysfunction. Furthermore, a portable scanner can help medical personnel to estimate the height and/or relaxation of the bladder neck for pelvic floor muscle training, assist in the efficacy evaluation of muscarinic antagonists in patients with neurogenic detrusor overactivity, and even determine whether the urine in the bladder of pediatric patients meets the criteria for catheterization to obtain urine samples. The National Institute for Health and Care Excellence (NICE) developed a MedTech innovation briefing (MIB) on the BladderScan BVI 9400 3D portable ultrasound scanner for the measurement of bladder volume (11). Portable bladder scanning was also recommended for the assessment of residual urine volume by the European Association of Urology (12,13).

In certain special cases, the results obtained with a portable bladder scanner can be affected by factors, such as dysuria accompanied by ascites, which is reported in the present case. The residual urine volume detected with the scanner was $560 \mathrm{~mL}$, but the actual urine output was only $120 \mathrm{~mL}$ (true urine volume); the difference between the two measurements was more than 4 -fold. This result indicates that the residual urine volume detected by the scanner was inaccurate, which led to incorrect, advanced timing of catheterization during management with intermittent catheterization. Previous studies have shown that bladder management is required by 1 million patients in the UK each year (14), and for approximately $25 \%$ of these patients, catheterization is unreasonable $(15,16)$. As described above, a portable bladder scanner is suitable for guiding intermittent catheterization, making a reasonable bladder management plan, and determining the correct timing of catheterization. However, in the present case, the residual urine volume was falsely increased by the presence of ascites, resulting in advanced timing of catheterization. Ideally, the volume of each urine output should be controlled at $400-500 \mathrm{~mL}$. If any interfering factors are not discovered in a timely manner, then the timing of catheterization based on the results of the scan could be advanced by mistake. As a consequence, the number of catheterizations would be unnecessarily increased, and the risk of the patient developing urinary tract infection might also be increased.
We consider the abnormal measurement results in the present case to be associated with the working principle of the portable bladder scanner. First, when measuring the bladder urine output, the ultrasound sensor is placed on the patient's lower abdomen (suprapubic area). The ultrasonic transducer generates sound waves and can receive sound waves reflected back from the bladder, thereby generating information for multiple cross-sectional scans. The scanning information is sent to the device computer, which automatically calculates and displays the capacity of the bladder. However, when using the device, it is extremely difficult to completely distinguish fluid located near the bladder and in the surrounding area from urine, which can result in superimposition of ascites and urine echoes and the inclusion of the ascites volume in the total residual urine volume. Ultimately, the computer calculations deviate massively from the actual measurement.

Although portable bladder scanners have been widely used in clinical practice, they cannot replace the clinical application of the catheterization assay entirely. Also, the staff using a portable bladder scanner might include clinicians or nurses rather than professional radiologists. If data are obtained based on bladder scanning alone and not imaging or the patient's actual condition, then the bladder fluid calculation might be inaccurate. Because the portable bladder scanner can reduce the workload of the operator, it takes about 2 minutes to measure a patient's bladder urine volume, and the machine can automatically identify, read data and generate reports. For patients, it is completed in the ward, which reduces the trouble of waiting in the B-ultrasound room, facilitates the patients to measure the urine volume at any time, and reduces the transportation and the occurrence of risk accidents for critically ill patients. Therefore, it is convenient and time-saving in clinical application. At present, there is no certificate requirement for the operator of portable bladder scanner, but it can be used after operation training. Therefore, in the future work, we may need to carry out short-term training classes for learning. The bladder scanner operator must not only master the operating technique but must also have a certain knowledge of ultrasound imaging to make a correct judgement based on the patient's condition rather than blindly relying on the instrument calculation.

A search of the literature in the China National Knowledge Infrastructure (CNKI) and Wanfang databases showed that, so far, relevant Chinese studies have been limited to the possibility, efficacy, and accuracy of applying portable bladder scanners in different diseases and medical 
fields; our case is the first report concerning their diagnostic limitations in China. As discussed, it is reasonable to infer that there are similar cases in clinical practice. When using a scanner to measure residual urine volume, an abnormal increase in total urine volume can occur, which may be attributable to complications, such as cysts in the pelvic cavity (17). Other studies have shown that when the urine volume is less than or equal to $150 \mathrm{~mL}$, the data measured by bladder scanner will be significantly higher than the actual urine volume (18). Some articles have also pointed out that flat bladder and a large prostate may hind accurate measurements (19) (flatted bladder and a large prostate may hind accurate measurements) also play an important role, (bladder shape plays a critical role in accuracy) (20). Regarding the clinical value of using a portable bladder scanner, medical personnel could collect specific cases in which the scanner interfered with the diagnostic results and summarize the findings to constantly improve its use. Currently, there are no operation guidelines for portable bladder scanners in China. Due to the high incidence and variability of dysuria, we are eager to develop guidance for portable bladder scanners to clarify the applicable populations, contraindications, and precautions, which can guide medical personnel in their more appropriate and reasonable use.

\section{Conclusions}

This case provides medical personnel with the following important information. First, a portable bladder scanner and catheterization can both be used to assess of residual urine volume. Second, when an abnormal residual urine volume is found using a portable bladder scanner, catheterization should be promptly applied to further confirm the diagnosis. Third, the interval and frequency of micturition or catheterization were evaluated according to the volume of body fluid. Attention should be given to the urination diary of the patients in order to rationalize their fluid intake of the patients and ensure that urine generation is regular. For patients with normal bladder sensory function, they should listen to the main complaints of patients carefully, and combine with abdominal palpation. Finally, when there is a large discrepancy between the scanner detection results and the actual measurement, healthcare professionals should look for pathological abdominal and pelvic problems to avoid diagnosis of a false increase in residual urine volume. In summary, situations similar to the case described herein are likely to be encountered in clinical practice. Patients with abnormal deviations in portable scanning results should not only be treated but should be further evaluated based on their condition; a failure to do this may lead to missed diagnosis of abnormal results and a delay in the optimal treatment time.

\section{Acknowledgments}

Funding: This study was supported by funding from General Research Projects of the Department of Education of Zhejiang University of China (grant No. Y201738128).

\section{Footnote}

Reporting Checklist: The authors have completed the CARE reporting checklist. Available at https://dx.doi. org/10.21037/tau-21-444

Conflicts of Interest: All authors have completed the ICMJE uniform disclosure form (available at https://dx.doi. org/10.21037/tau-21-444). The authors have no conflicts of interest to declare.

Etbical Statement: The authors are accountable for all aspects of the work in ensuring that questions related to the accuracy or integrity of any part of the work are appropriately investigated and resolved. Consent for the publication of this case report was obtained from the patient's relatives. All procedures performed in studies involving human participants were in accordance with the ethical standards of the institutional and/or national research committees and with the Helsinki Declaration (as revised in 2013).

Open Access Statement: This is an Open Access article distributed in accordance with the Creative Commons Attribution-NonCommercial-NoDerivs 4.0 International License (CC BY-NC-ND 4.0), which permits the noncommercial replication and distribution of the article with the strict proviso that no changes or edits are made and the original work is properly cited (including links to both the formal publication through the relevant DOI and the license). See: https://creativecommons.org/licenses/by-nc-nd/4.0/.

\section{References}

1. Baumann BM, Welsh BE, Rogers CJ, et al. Nurses using volumetric bladder ultrasound in the pediatric ED. Am J 
Nurs 2008;108:73-6.

2. Kim HJ, Chun MH, Han EY, et al. The utility of a bladder scan protocol using a portable ultrasonographic device in subacute stroke patients. Disabil Rehabil 2012;34:486-90.

3. Rigby D, Housami FA. Using bladder ultrasound to detect urinary retention in patients. Nurs Times 2009;105:34, 36-7.

4. Newman DK, Smith DA. A portable bladder scanner. Nurse Pract Forum 1991;2:243-5.

5. Okamoto H, Murakami N, Carvajal CC, et al. Positional uncertainty of vaginal cuff and feasibility of implementing portable bladder scanner in postoperative cervical cancer patients. Phys Med 2018;45:1-5.

6. Liu S, Xu F, Xu T, et al. Evaluation of Vesical ImagingReporting and Data System (VI-RADS) scoring system in predicting muscle invasion of bladder cancer. Transl Androl Urol 2020;9:445-51.

7. Moselhi M, Morgan M. Use of a portable bladder scanner to reduce the incidence of bladder catheterisation prior to laparoscopy. BJOG 2001;108:423-4.

8. Ung KA, White R, Mathlum M, et al. Comparison study of portable bladder scanner versus cone-beam CT scan for measuring bladder volumes in post-prostatectomy patients undergoing radiotherapy. J Med Imaging Radiat Oncol 2014;58:377-83

9. Kuppusamy S, Gillatt D. Managing patients with acute urinary retention. Practitioner 2011;255:21-3, 2-3.

10. Cathcart P, van der Meulen J, Armitage J, et al. Incidence of primary and recurrent acute urinary retention between 1998 and 2003 in England. J Urol 2006;176:200-4; discussion 204.

11. Mulder FE, Schoffelmeer MA, Hakvoort RA, et al. Risk factors for postpartum urinary retention: a systematic review and meta-analysis. BJOG 2012;119:1440-6.

12. Chaurasia A, Tyagi K. Persistent postpartum urinary retention following vaginal delivery: a rare complication

Cite this article as: Zhu X, Zou L, Yao Z, Chen Z. Abnormal deviation in the measurement of residual urine volume using a portable ultrasound bladder scanner: a case report. Transl Androl Urol 2021;10(7):3084-3088. doi: 10.21037/tau-21-444 in obstetrics practice. Int J Reprod Contracept Obstet Gynecol 2013;2:475-7.

13. Luo H, Wang Y, Li F, et al. Comparative study on evaluating the bladder volume between BladderScan BVI9400 and Ultrasound System iU22. Zhongguo Yi Liao Qi Xie Za Zhi 2015;39:295-8.

14. Nazarko L. Effective evidence-based catheter management: an update. Br J Nurs 2010;19:948, 950, 952-3.

15. Fakih MG, Pena ME, Shemes S, et al. Effect of establishing guidelines on appropriate urinary catheter placement. Acad Emerg Med 2010;17:337-40.

16. Rothfeld AF, Stickley A. A program to limit urinary catheter use at an acute care hospital. Am J Infect Control 2010;38:568-71.

17. Alagiakrishnan K, Valpreda M. Ultrasound bladder scanner presents falsely elevated postvoid residual volumes. Can Fam Physician 2009;55:163-4.

18. Yang YH, Chen CY. Accuracy of residual urinary volume measurements in patients with neurogenic bladder when using a portable ultrasound bladder scanner. Taiwan Journal of Physical Medicine and Rehabilitation 2018;46:63-9.

19. Yamaguchi Y, Kamai T, Kobayashi M. Comparative accuracy of the Lilium $\alpha-200$ portable ultrasound bladder scanner and conventional transabdominal ultrasonography for postvoid residual urine volume measurement in association with the clinical factors involved in measurement errors. Neurourol Urodyn 2021;40:183-92.

20. Zhao L, Liao L, Gao L, et al. Effects of bladder shape on accuracy of measurement of bladder volume using portable ultrasound scanner and development of correction method. Neurourol Urodyn 2019;38:653-9.

(English Language Editor: J. Reynolds) 